\title{
Experimental Biologist Perspective on Inhabiting Other Worlds
}

Kass L*

School of Biology \& Ecology, University of Maine, USA

*Corresponding author: Leonard Kass, School of Biology and Ecology, University of Maine, Orono, ME 04469 USA, Email: leonard.kass@maine.edu

\section{Editorial \\ Volume 2 Issue 6}

Received Date: November 04, 2019

Published Date: November 11, 2019

DOI: $10.23880 /$ izab- 16000184

\section{Abstract}

Humans and Earth's entire biota will become extinct if they do not inhabit another world. This transference will require substantial support, long-term planning, S.T.E.M. and social applications, along with more clear-eyed determination than humanity has yet exhibited. Some of these more difficult challenges involve changing the way humans view themselves. This introspection will include honest assessments of our biological limitations as well as our dependence upon other life-forms. We will need to redefine ourselves as a family and act decisively upon that new definition. This work outlines a difficult yet realistic approach towards our long-term survival as a species, the only one on Earth most capable of preserving its biota and itself. The conclusions deduced here are contrasted with current concepts on this topic that do not adequately incorporate biology as it has evolved for billions of years on Earth. The perspective expressed within may appear radical when comparing it to alternate viewpoints that do not adequately incorporate biology, its evolution, and its limitations.

Keywords: NASA; Tarian Criterion; Humanity

\section{Current Concepts on Inhabiting Other Worlds}

Practically all conventional writers of fiction and science assume that humans will someday establish longterm habitations on moons or other planets [1]. By longterm, I mean thriving from generation to generation with reproductive success. It is likely that this otherworld human emigration is presumed because thinking otherwise would consign humanity, along with all other life on earth, to biological extinction. Humans must transfer to other worlds because modern science has established that the Earth will become untenable for life: Our sun will die or turn into an enlarged Red Giant [2]. We either emigrate or become extinct along with all other life forms remaining here on Earth. How much longer humanity survives on Earth is a matter of conjecture and differing opinions. Some eminent scientists calculate that Earth will be inhabitable in another billion years, while others think humanity will not survive the next several hundred-years [3-5]. Although established scientists differ dramatically on this time scale for human survivorship on Earth, all do agree that humanity must vacate Earth sooner or later, to avoid extinction. But where to go, and how to get there?.

Two of the most popular hypothetical targets for human extraterrestrial habitations are the moon and Mars. The moon has an advantage of being most proximal to Earth, but disadvantage of appearing very alien with its 


\section{International Journal of Zoology and Animal Biology}

absence of atmosphere. In contrast, color pictures Mars make it appear as though one could wander around on its surface as if in the Mojave Desert [6].

\section{Practical Problems with Current Concepts of Inhabiting Other World}

From an experimental biologist perspective, there are numerous problems with the current consensus view concerning human habitations on other worlds. Perhaps Elon Musk with his SpaceX program is unaware of these issues as they advocate and fund an attempt to secure a colony on Mars within the next 20 years [7]. NASA too has made one of its missions the creation of habitats on the moon or Mars [8]. Besides the moon or Mars, there are no serious plans for inhabiting other worlds within our solar system. Humans briefly visit the International Space Station. However, it requires constant infusion of resources, energy, and new resources. Long-term human habitation must be independently self-sustaining. Only certain planets and moons might provide those resources.

There are problems of a biological nature that are terminal to habitats on moons and planets. Scientists have been speculating living in and traveling through space for many years. Scientists are becoming ever more aware of serious health issues associated with living under microgravity for even brief periods. In 2019, NASA published the Twin Study [9] that has also summarized the deleterious effects related to life in space. The more critical maladies that occur after just weeks or months in space are reported to include physiological pathologies likely to lead to blindness over time: optic disk edema, hyperoptic shifts, globe flattening, cotton wool spots, and choroidal folds. Vascular physiological changes include "puffy face" and "bird leg" syndrome caused by vessel distensions and about 2 liters of interstitial fluid displacements. Serious cognitive declines were also verified that may be permanent and accumulative. This 20-page Science publication, and other studies it references, uncovered various other grave concerns of a biological nature that includes chromosome telomere elongations, gene instabilities, transcriptional and metabolic changes, and DNA methylation changes in the immune and [9]. Although these are known space-travel human pathologies that occur under zero or micro-gravity conditions, we should anticipate other serious medical issues under gravitational conditions that differ significantly from Earth's constant $1.0 \mathrm{~g}$ gravitational force if multigenerational habitations are taken into consideration. In comparison to Earth's, the moon has $1 / 80^{\text {th }}$ the mass and gravity of $0.17 \mathrm{~g}$. Mars has a mass of $1 / 10$ of the Earth and a gravity of $0.38 \mathrm{~g}$. Unfortunately, neither the moon nor Mars will support human health through even a single new generation of human pioneers. Based upon what we know about human physiology, no scientist should predict that pregnancy, fetal development, birth, infancy, childhood, and maturation to adulthood would survive conditions differing significantly from a gravity of $1 \mathrm{~g}$. This fact should not come as a surprise, as all life on Earth evolved on a planet of $1 \mathrm{~g}$. All physiological processes have depended upon this unvarying condition over its billions of years of evolution. In our entire solar system, there is only one planet or moon that has a gravity of nearly $1 \mathrm{~g}$ other than Earth: Venus. The reason no one has seriously suggested that humans attempt to live on (or below) the Venusian surface is due to its very highly acidic, corrosive and lethal atmosphere. In addition, as the sun converts into a Red Giant, Venus will be second in line for destruction. It follows from the above that humans will never inhabit in a multi-generational manner any of the worlds within our solar system, other than Earth. This is such a sobering and painful conclusion that others may feel a need to create various arguments to dispute this conclusion. There is, indeed, a solution to these physical and biological constraints: I will describe an alternate course of action for our long-term survival in the following section. However, its adaption by us will require humanity to redefine itself and our relationships to other life forms. If humanity is unable or unwilling to make the necessary behavioral changes, science and logic suggest no alternate route around terrestrial extinction. I then list commonly believed alternatives to the pathway proposed and show how each, in turn, are unreasonable within the physical and biological limits in which we find ourselves on Earth and within our solar system. An experimental biological perspective will prove useful for understanding the tenets underlying these arguments.

\section{Solving Four Major Problems in Inhabiting Worlds outside our Solar System}

In spite of the numerous practical problems involved, there is at least one viable solution that would allow for permanent human habitations on other worlds. As no moon or planet in our solar system will support longrange plans for a new home, we must seek suitable worlds with a mass about that of the earth in other star systems. There are innumerable star systems that will be suitable. The following are the four general criteria for successful interstellar space travel and subsequent human habitation. First, its launch must solve the physical constraints imposed on any and all successful interstellar space travel. Second, its launch and travel must contain components consistent with its serious energy constraints. Third, during its flight, and also upon arrival, the space 


\section{International Journal of Zoology and Animal Biology}

craft must contain something of sufficiently high general intelligence and perseverance to solve unforeseen technical and behavioral problems involved in establishing new habitats for humanity. Finally, the intelligence carried on board must genuinely want human societies to thrive independently on these distant worlds in a self-sustaining manner. I refer to this constraint as the Tarian Criterion. In the following paragraphs, I justify these four criteria needed to establish human habitats on new distant worlds.

The physical constraints imposed upon any such travel from Earth to a distance star are sobering and include mass and robustness. Today, it costs $\$ 5,000$ USD to put a Kilogram (Kg, about a half-pound) of payload into Earth orbit, though NASA hopes to get the price down several orders of magnitude within the next 40 years [10]. Whatever that cost ultimately becomes, it will still represent a small fraction of the cost of propelling a single $\mathrm{Kg}$ to $10 \%$ the speed of light (0.1c) from Earth's orbit to a nearby star. One-tenth $\mathrm{c}$ is anticipated so that the travel towards the nearest star would take less than a century in travel time. Whatever that interstellar travel cost calculation becomes, it must be doubled for the deacceleration necessary for planetary orbit and other actions upon arriving at its destination. The craft will need to carry a source and housing for this energy source on board for this braking power. This too will add necessary mass that will need to be propelled. The point made here is that any mass sent will be at an extremely high cost premium for this interstellar space travel. In addition to mass, another physical constraint is that the cargo and ship (assuming the cargo will require a protective shell) must be extraordinarily robust to survive travelling through outer space for at least a century. Robustness is necessary, not only for the interstellar travel itself at extraordinarily high speeds, but also for the star or planetary orbiting likely needed for repairs and replenishing energy reserves required to continue its mission. The cargo and craft will need to remain functional for a century or longer, until new world factories are established for needed repairs and buildings. To experimental biologists and others of like minds, it becomes obvious that humans will not themselves travel outside our solar system. Human bodies are mostly made of water, which would represent unnecessary and wasteful mass to transport. Humans would require other associated mass needed for their protection and survival over the centuries in space. Human bodies, particularly their brains, are not robust. Human organs are fragile.
The energy constraints imposed on space travel are as severe, perhaps more so, than physical. For all accelerations, deaccelerations, and non-gravitational directional changes, a sizable energy source will need to be carried onboard as part of its cargo constraint at great energy cost. This onboard energy source and its requisite mass, for its storage and conversions, will need to be kept at a minimum with only adequate onboard energy to propel, break, and employ photoelectric panels to collect energy from the new star once an orbit has been accomplished. To experimental biologists and others, humans will not, themselves, travel anywhere in space when energy constraints are in play. Humans require temperatures of about $293^{\circ} \mathrm{K}\left(20^{\circ} \mathrm{C}\right.$ or $\left.68^{\circ} \mathrm{F}\right)$. Interstellar space is at about $3^{\circ} \mathrm{K}$. Any living quarters for humans would need to be continuously heated $290^{\circ} \mathrm{K}$ for centuries until a habitable ecosystem has been re-established on the new world. Even, as science fiction (SciFi) would have us think that the human body could be refrigerated down to $273^{\circ} \mathrm{K}\left(0^{\circ} \mathrm{C}\right.$ or $\left.-32^{\circ} \mathrm{F}\right)$, it would be too expensive. No reputable experimental biologist would think the human body could be subjected to temperature much below that freezing limit for any significant length of time (e.g. years), without lethal damage. The point made here is that humans will not, themselves, travel outside our solar system and remain alive. If humans do not engage in interstellar travel, then what will on our behalf?

Now that we have established that no human body will travel between stars, the third criterion for successful interstellar travel, and establishment of new human habitation, is that something contained within the cargo of the space ship must possess superior and artificial general intelligence (SAGI, related to "Seed AI" [11], with capabilities of self-improvements in $\mathrm{AI}$ ) so as to be capable of solving or correcting any unforeseen difficulty very likely to arise. I assume this SAGI to be at, and most likely far above, human level intelligence (HLI) [12]. This SAGI unit, or units (since redundancy is an important engineering and biological necessity) must not only be capable of solving tactical and engineering challenges, but have the social and psychological capacities of raising humans from birth to adulthood in a normal (at least by contemporary Earth standards) affectionate and protective environment. These entities would someday need to assume human form so as to become an integral part of these growing family units for at least several generations of humans before modeling age-related afflictions, death, and moving on. Through these means, an entirely new human society could be raised, human parental behaviors modeled, behaviors of aging generations modeled, for their human "children" and 


\section{International Journal of Zoology and Animal Biology}

families. Establishing this new and productive human society would be the end point toward social sustainability on this new world. Certainly, to accomplish all of the above would minimally require SAGI of some sort. Note that the SAGI envisioned could best satisfy the demands of criteria 1 and 2 above for interstellar travel using minimal mass and energy while allowing for maximum robustness in $\mathrm{AI}$ design.

The fourth and final requirement, named the Tarian criterion, for successful interstellar travel and human habitation on a new world follows naturally from those three other considerations described. The SAGI units must genuinely want to have humanity exist and thrive on new worlds. This Tarian criterion becomes obvious when one considers that it might take such SAGI units a century to travel from one particular star system to the next. It would likely take hundreds of millennia to recreate a significant part of Earth's biota within, I presume, a large underground ecologically balanced and sustainable terrarium established by these SAGI units before the rearing of human offspring could be reasonably contemplated. Populating this stable biota with the new human inhabitants in isolated and sealed underground chambers provide radiation and biocontamination barriers. Experimental biologists will want to prevent any possible cross-contaminations between existent native life-forms with those introduced from Earth: Cohabitations on worlds with HLI beings would not be allowed. These SAGI units would need to take great interest in and dedication for humanity's survival and establishing its society. Such SAGI units would need to persevere in these responsibilities under the harshest of conditions for millennia. But this requisite dedication is no more than a human parent would be expected to do for their child and grandchildren if needed and capable of such challenge. Humans have biological drives to bear children and to support, care, and love their offspring as they might other humans. These Tarian SAGI units will not be biological in nature and would not have evolved such emotional drivers as have humans. To accomplish all they would need in starting new human societies on other worlds they would also need to be sentient, conscious and self-aware. What then would be their motivation for such attention, dedication, care, and perseverance over long times, with resource allocation towards humanity's future? I address this topic in the next section.

While discussing the need for the Tarian criterion in establishing new world habitations for humans outside our solar system, I outlined some essential attributes of these complex special function SAGI units. Since these specific characteristics and others below, have not before been conceptualized in fictional, scientific, nor philosophical works, it will prove useful to provide them a name: "Tarian". As conceptualized, these Tarians, are the ultimate humanitarians. They are sentient by which I mean they possess self-awareness or consciousness at least approximately on the level with H. sapiens sapiens. "Humani" could be translated as "from human" and the Tarians, as proposed here, are envisioned as having been derived from or created by humans at some point in our future. Other candidate names for these entities carry with them misleading and unwanted connotations. The word "robot" is derived from enforced laborer or slave, which Tarians are not. The word "android" is derived from "male" and generically for robots taking on human forms. Tarians would assume human form only when necessarily interacting with humans. Those times would be relatively rare. The word "cyborg" connotes something part metal machine and biological [13]. Tarian is a new name for a new concept of SAGI sentient being designed for specific purposes and necessities as described here for the first time. The next section justifies the rationale, and even necessity, for imbuing our interstellar space humanitarians with the characteristics ascribed to these Tarians.

\section{Greatest Challenges Ahead are Biological Not Technological}

The greatest challenges confronting humanity today, to avoid extinction that has happened to over $99.9 \%$ of all species that once lived on Earth, are biological in nature and not technological. The human brain, the most complex compact structure of intelligence evolved in the known universe, is composed of units that came to life on Earth over billions of years. Physical laws were exclusively followed during this non-directional evolutionary process. Our brain is composed of cellular units and their biological products, as is the rest of the human body. Neurobiology is gaining insights into how the human brain works in providing processes related to thought, creativity, consciousness, and emotions. The human brain and its properties are not magical. There is no known theoretical limit on constructing a SAGI out of non-biological materials, such as solid-state circuits, capable of deep learning, emotions, self-awareness and consciousness. To think otherwise about the structure and function of the human brain would be mystical thinking, contrary to that adapted by experimental biologists and other like-minded individuals. Indeed, we assume that future SAGI units will assist us in constructing newer versions of them with evermore advanced SAGI characteristics (i.e. Seed AI) until they far 


\section{International Journal of Zoology and Animal Biology}

surpass $H L I$ in abilities to think more deeply and creatively. Humanity will then have reached the so-called "singularity" [14]. The greatest challenges, by then, clearly become biological, and include psychological, behavioral, and social changes within ourselves. The question then becomes not whether we can design and construct these Tarian SAGI units with strong drives to affiliate themselves with humanity, but whether humans will place strong value into doing so. The values that humans place on their various behaviors, present and future, have biological origins.

Although the human body is fragile, neural functions in some humans exhibit remarkable flexibility. Humans can form close and strong family-level affections and love to not closely related humans and even cats, dogs, and other animals. Placed into a warm and fuzzy package, a Tarian in the form of a baby or child, could be loved by humans far more than are Furbies [15]. Promoting strong bond formations between Tarians and their surrogate human parents, siblings and friends would be straightforward if the biology of humans was better understood. The science of affection and love, in either direction, should inform the requisite architecture, programming, and behavior. More biological research in areas of human psychology is needed to understand and disable selfishness, greed, fear, xenophobia and immediate gratification. Understanding more biology would be useful today in promoting better communities, societies and environments on Earth. It will be critical in dealing fairly and equitably with Tarians or any sentient SAGI unit. Learning how best not to misuse fellow humans would be a good start. Large-scale modifications in human behavior are needed to improve societies and the global environment, now and into our distant futures. Fortunately, an enlightened and small community could raise a few Tarians and imprint the best that humanity has to offer onto this adopted family. Most progress in human societies have occurred through efforts of just a relatively few dedicated individuals [16].

Human societies, along with much of Earth's biota, can thrive together in other star systems with the help of Tarians. These special SAGI units and would need do no more than that expected from caring human parents, or that expected from caregivers of humans who are otherwise unable to properly care for themselves. Humans and our ancestors have successfully accomplished this feat for millions of years. One key to this altruism is to consider others as family. Contemporary neuroscience does not fully understand the neurocircuitry and brain processes underlying affection, dedication, loyalty, or love. Once understood, it can be emulated by SAGI. Can we, as humans, genuinely expand our concept of family to these sentient Tarians? If they were imbued with the capabilities of emulating familial love, would humans, in turn, be sincere in our reciprocity? Once built and functional, would we share equal treatment under the law as we would any family member? How they might be greeted and treated by other humans is the crux of the biological problem. Would we fear and reject our own children if they were an order of magnitude or more intelligent than we? The answer to that question might depend upon whether humans treat them respectfully, provide for them to our own material detriment, and genuinely care for them as they grow. Hopefully, the selected parents or teachers of these new Tarians infuse them with as much resilience, forgiveness, aspiration, and affection as is humanly possible and as we attempt with our biological children. Let us also hope our Tarians inherit the perspectives of the experimental biologist: No different species is inferior to another. There is no known physical law preventing us from constructing Tarians, SAGI that could return genuine affection. But will human behavior become adaptable enough to accomplish that feat, even in a minority? Many computer engineers and scientists are convinced that it is just a matter of time before SAGI units become a reality. When that happens, unlike humans, these SAGIs can and will make themselves more and more intelligent, unimpeded by processes inherent to biological evolution [12]. In addition, we may need Tarians to save humanity from amoral SAGIs some might create [17]. A new solution to the Fermi Paradox (i.e. Why is not the galaxy already filled with space-faring aliens? [18]), arising from this perspective, is that other $H L I$ species evolved on other worlds were not able to overcome their specie-ism but hubris (i.e. biology) in time to prevent ever more intelligent and non-affiliated SAGIs from taking over worlds. Creating Tarians, accepting them as family, and imbuing them with the best ethics humanity can offer may be the only way for our families and Earth's biota to survive and thrive into the distant future.

\section{Conclusion}

In this short discourse I have outlined the challenges facing humanity today if it is not to become extinct in the near or distant future. I list four criteria for interstellar space travel and subsequent human habitations on distant worlds that requires the creation of special SAGI units named Tarians. I describe the attributes and designed purpose of these Tarians. It remains to be seen whether we as a species can rise above hubris, conceit, 


\section{International Journal of Zoology and Animal Biology}

and selfishness in accepting other life forms as family. This question, as well as others biological in nature, will determine our near and long-range survival.

\section{Acknowledgement}

The author thanks Drs. Janice L. Pelletier, Richard Kass, and Michael Wieder for providing many thoughtful and important suggestions.

\section{References}

1. Wall M (2018) Out There: A Scientific Guide to Alien Life, Antimatter, and Human Space Travel (For the Cosmically Curious) Grand Central Publishing pp: 256.

2. Scudder J (2015) The sun won't die for 5 billion years, so why do humans have only 1 billion years left on Earth?.

3. Powell C (2019) Cyborgs will replace humans and remake the world, James Lovelock says.

4. (2014) Hawking: AI could end human race. BBC.

5. Sparkes M (2015) Top scientists call for caution over artificial intelligence.

6. (2019) Scientists Simulate Moon and Mars Exploration in Mojave Desert.

7. (2019) Elon Musk.

8. (2019) Explore Moon to MARS.
9. Garrett Bakelman, Darshi M, Green SJ, Gur RC, Lin L, et al. (2019) The NASA Twins Study: A multidimensional analysis of a year-long human spaceflight. Science 364(6436).

10. (2019) Advanced Space Transportation Program: Paving the Highway to Space accessed.

11. Yudkowsky E (2001) General Intelligence and Seed AI-Creating Complete Minds Capable of Open-Ended Self-Improvement.

12. Kass L (2018) K-Conjecture - The Future of Biology. Int J Zoo Animal Biol (5): 000126.

13. (2019) Robot.

14. S Ulam (2019) Tribute to John von Neumann. pp: 149.

15. Marsh A (2019) Coded for Cuteness: How the Furby Conquered Hearts and Minds. Real programming chops lay behind the Furby's cuddly, creepy façade.

16. Fallows J (2013) The 50 Greatest Breakthroughs Since the Wheel. The Atlantic.

17. Yudkowsky E (2019) Concise Summary Singularity Institute for Artificial Intelligence.

18. Woodward A (2019) The Fermi Paradox asks why we haven't found any evidence of aliens. Here are 13 potential answers to that question. Insider Inc. 